\title{
Absolute cross sections for electronic excitation of furan by electron impact
}

\author{
Khrystyna Regeta and Michael Allan \\ Department of Chemistry, University of Fribourg, chemin du Musée 9, CH-1700 Fribourg, Switzerland
}

(Received 21 November 2014; published 12 January 2015)

\begin{abstract}
We measured differential cross sections for exciting the three lowest electronically excited states in furan, as functions both of electron energy and of scattering angle. Emphasis of the present work is on recording detailed excitation functions, revealing resonances in the excitation process. The cross section for the first triplet state has a shoulder in the first $2 \mathrm{eV}$ above threshold, assigned to the high-energy tails of the shape resonances, followed by two peaks at 6.5 and $8.0 \mathrm{eV}$, assigned to two ${ }^{2}\left(\pi, \pi^{* 2}\right)$ core-excited shape resonances with substantial configuration mixing. The excitation mechanism thus closely follows that of the prototype case of ethene, except that as the number of double bonds doubles, the numbers of both shape and core-excited resonances also double. The cross section for the singlet states around $6 \mathrm{eV}$ (primarily the $S_{2}{ }^{1} B_{2}$ state) rises linearly with energy and attains very high values in the forward direction as expected for a dipole-allowed transition, but has a vertical onset at higher scattering angles, possibly due to a threshold core-excited ${ }^{2}\left(\pi, \pi^{*} 3 \bar{s}\right)$ resonance responsible also for dissociative attachment. Elastic cross sections are also presented. Both elastic and inelastic absolute values compare favorably with existing measurements where available, except very near threshold. The results are compared to existing calculations.
\end{abstract}

\section{INTRODUCTION}

Electron-driven chemistry of polyatomic molecules is finding an increasing number of applications, including radiation therapy, plasma-assisted chemical vapor deposition, and focused electron-beam-induced processing (FEBIP). Electronic excitation by electron impact is often the first step for neutral dissociation and thus an alternative to the more commonly studied dissociative electron attachment. Whereas dissociative electron attachment has been recently studied for biomolecules and molecules relevant for FEBIP, studies of electronic excitation of polyatomic molecules are much less numerous. The present study extends our past effort in this direction, which started by the studies of ethene [1,2]. Furan is particularly suited as a prototype for the next step towards higher complexity, having one more $\mathrm{C}=\mathrm{C}$ bond than ethene, but still is simple enough to be amendable to precise $a b$ initio calculations.

An important study of electronic excitation of furan by electron impact, both theoretical and experimental, has recently been carried out by da Costa et al. [3]. That study has been preceded by a study of elastic scattering by the same theoretical and experimental groups [4]. Grand total cross section has recently been measured by Szmytkowski et al. [5].

Other work involving electron collisions with furan is the experimental study of electron-impact vibrational excitation by Hargreaves et al. [6], the theoretical study of the elastic cross sections by Bettega and Lima [7], the study of polarization effects on electronic excitation by da Costa et al. [8], and the study of dissociative electron attachment (DEA) by Sulzer et al. [9].

The present study concentrates on recording the cross sections for electronic excitation as a function of energy, revealing the resonant structure and permitting conclusions about the excitation mechanism. This continues the early effort of Asmis from this laboratory [10], who measured relative excitation functions at a fixed scattering angle (superposition $0^{\circ}$ and $180^{\circ}$ ) with a magnetically collimated electron spectrometer for furan but also for cyclopentadiene, thiophene, and pyrrole. He observed distinct resonant structures in the excitation functions which he assigned to core-excited shape resonances by qualitative arguments and semiempirical configuration-interaction (CI) calculations. For completeness and for comparison with earlier data, the present work also includes elastic cross sections and discusses the relation of the present results to DEA.

\section{ELECTRON IMPACT SPECTROMETER}

The measurements were performed using an electronimpact spectrometer described earlier [2,11]. It employs hemispherical analyzers to improve resolution, which was reduced to $22 \mathrm{meV}$ (in the energy-loss mode) for the present study to gain signal for the weak electronic excitation. The electron beam current was 300-800 pA. The energy of the incident electrons was calibrated on the $19.365 \mathrm{eV}^{2} S$ resonance in helium [12] and is accurate to within $\pm 10 \mathrm{meV}$. The sensitivity of the instrument is not constant when the electron energies are varied, but this effect, expressed as the "instrumental response function", was quantized on elastic scattering in helium and all spectra were corrected as described earlier [2,11]. The values of the cross sections were determined by the relative flow technique as described by Nickel et al. [13] using the theoretical helium elastic cross sections of Nesbet [14] as a reference. The confidence limits of the magnitudes are $\pm 15 \%$ for the elastic cross sections and $\pm 25 \%$ for the inelastic cross sections. The furan and helium pressures in the gas inlet line were typically 0.08 and 0.24 mbars, respectively, during the absolute measurements.

The incident electron energy, the energy of the scattered electron (the residual energy), and the electron energy loss are labeled $E_{i}, E_{r}$, and $\Delta E$, respectively. Note that electron energy-loss spectra can be recorded by holding either $E_{i}$ or $E_{r}$ constant and both types are presented in this paper. The "constant $E_{i}$ spectra" are used in Sec. IIIC to derive the absolute magnitudes of the inelastic cross sections, because 
the magnitudes are required at the same incident energies for various final states, for the purpose of the tables and the angular distributions. The "constant $E_{r}$ spectra," presented in Sec. III B, are more appropriate to characterize spectroscopy of the target. This is because the character of the excitation (i.e., spin and/or dipole forbidden, or allowed) depends primarily on how far above threshold the excitation happens, and "constant $E_{r}$ spectra" have the advantage of being recorded at the same energy above threshold across the entire $\Delta E$ range. This is particularly relevant for spectra recorded near threshold. The "constant $E_{r}$ spectra" further have the (minor) technical advantage of not requiring correction for the analyzer response function.

\section{RESULTS}

\section{A. Elastic scattering}

Angular distributions are shown in Fig. 1. As in our earlier work, the curves were obtained by scanning the magnetic angle changer, in steps of $2.5^{\circ}$, around the fixed analyzer positions of $20^{\circ}, 45^{\circ}, 90^{\circ}$, and $135^{\circ}$ (with some overlap providing consistency check) and then combining the segments into one curve. Absolute values were determined by the relative flow method at the scattering angles of $20^{\circ}, 45^{\circ}, 90^{\circ}, 135^{\circ}$, and $180^{\circ}$

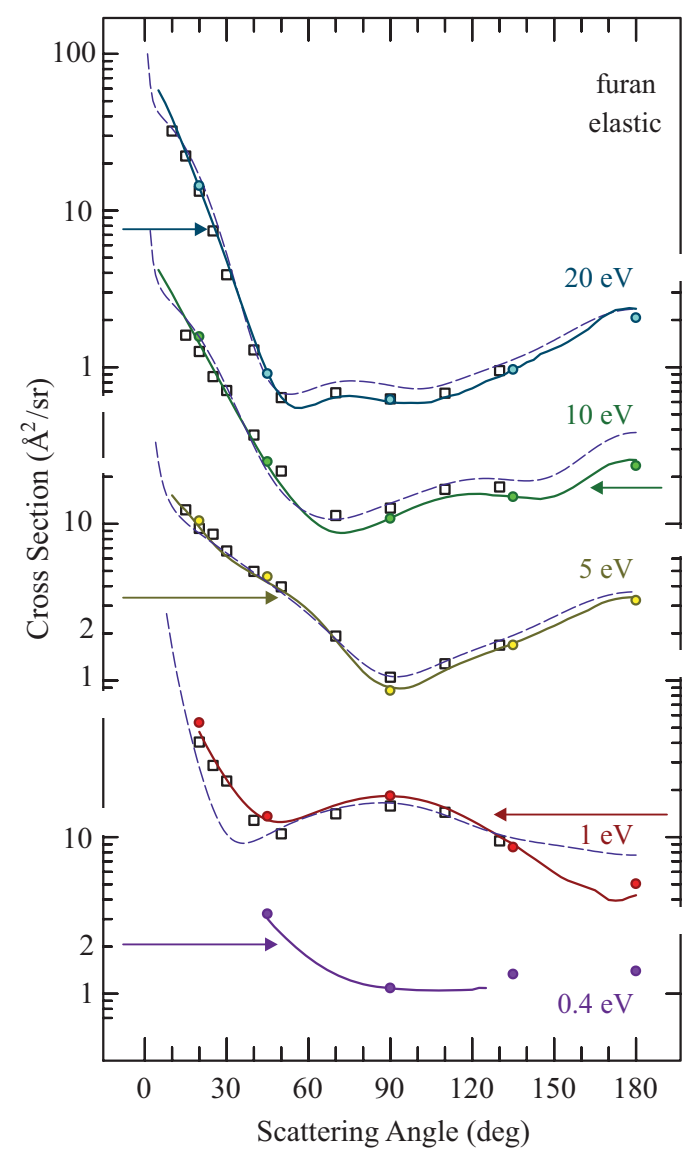

FIG. 1. (Color online) Angular distributions of the elastic cross section. Continuous lines and circles show the present data. Squares show the experimental data of Khakoo et al. [4], and dashed lines show the calculated cross sections of da Costa et al. [3]. Horizontal arrows indicate which scale belongs to which spectrum.
TABLE I. Integral elastic cross sections, in $\AA^{2}, \pm 20 \%$.

\begin{tabular}{lccccc}
\hline \hline Energy & $0.4 \mathrm{eV}$ & $1 \mathrm{eV}$ & $5 \mathrm{eV}$ & $10 \mathrm{eV}$ & $20 \mathrm{eV}$ \\
\hline Present & 21.9 & 20.3 & 32.6 & 32.2 & 26.0 \\
Exper. Ref. [4] & & 22.8 & 36.1 & 32.5 & 23.9 \\
Theory Ref. [3] & & 16.1 & 33.1 & 37.7 & 27.0 \\
\hline \hline
\end{tabular}

and the smooth curve was normalized to these discrete values, which are shown in Fig. 1 as circles - the redundancy provides a check of consistency. The agreement with the theoretical data of da Costa et al. [3] and experimental data of Khakoo et al. [4], both shown in Fig. 1, is very good. (Note that we take the experimental elastic data from the publication of Khakoo et al. [4], devoted to elastic scattering, but the theoretical data is from the later publication of da Costa et al. [3], which focuses on electronic excitation but also presents improved theoretical elastic data, which includes the effects due to multichannel coupling [15].)

Table I lists integral cross sections obtained by integrating under the angular distributions (with visual extrapolation in the forward direction). Given the very good agreement of the differential cross sections, the excellent agreement with the theoretical data of da Costa et al. [3] and experimental results of Khakoo et al. [4] is not surprising.

The elastic cross sections were then recorded as a function of electron energy as shown in Fig. 2. The effect of the two shape resonances (discussed in more detail below) is seen in the 1.5- to 4-eV range, in particular at $90^{\circ}$ and $135^{\circ}$. The agreement

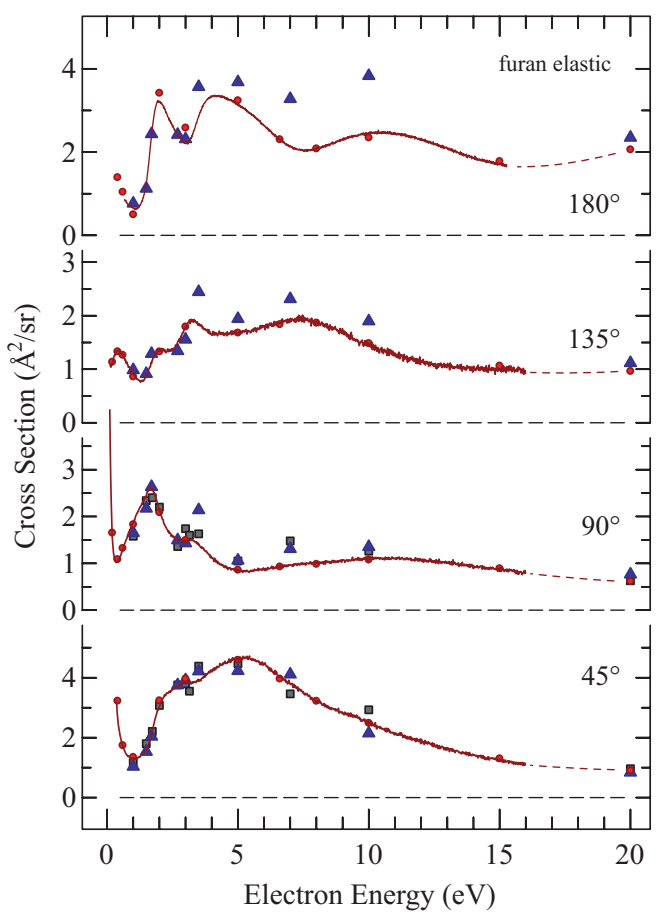

FIG. 2. (Color online) Elastic differential cross sections. Present data are shown as continuous red (gray) lines and circles, the calculated cross sections of da Costa et al. [3] are shown as triangles, and the experimental data of Khakoo et al. [4] are shown as squares (average of their $40^{\circ}$ and $50^{\circ}$ data is compared to our $45^{\circ}$ data). 
with the calculated cross sections of da Costa et al. [3] and the experimental data of Khakoo et al. [4] is very good, although the differences are more magnified on the linear scale than they were on the logarithmic scale in Fig. 1.

\section{B. Energy-loss spectra}

Electron energy-loss spectra were recorded to characterize the excited states for which the excitation cross sections were measured. Representative spectra emphasizing forbidden and allowed transitions are shown in Fig. 3. The positions of the bands agree with earlier spectra of Flicker et al. [17], Palmer et al. [18], Khakoo et al. [3], and Giuliani and Hubin-Franskin [19]. The spectrum recorded at $0^{\circ}$ agrees well with the vacuum-ultraviolet (VUV) spectrum of Palmer et al. [18].

For the purpose of measuring the cross sections we divided the energy-loss range 3.25-6.55 eV into three sections (3.254.85, 4.85-5.59, and 5.59-6.55 eV), indicated in Fig. 4, and integrated the signal within them. Our cross sections refer to all excitation processes within each range. The cross sections for the $T_{1}$ and $T_{2}$ states do not suffer appreciably from band overlap, but the third section contains four singlet states so that although the symbol $S_{2}$ is used to designate it and although

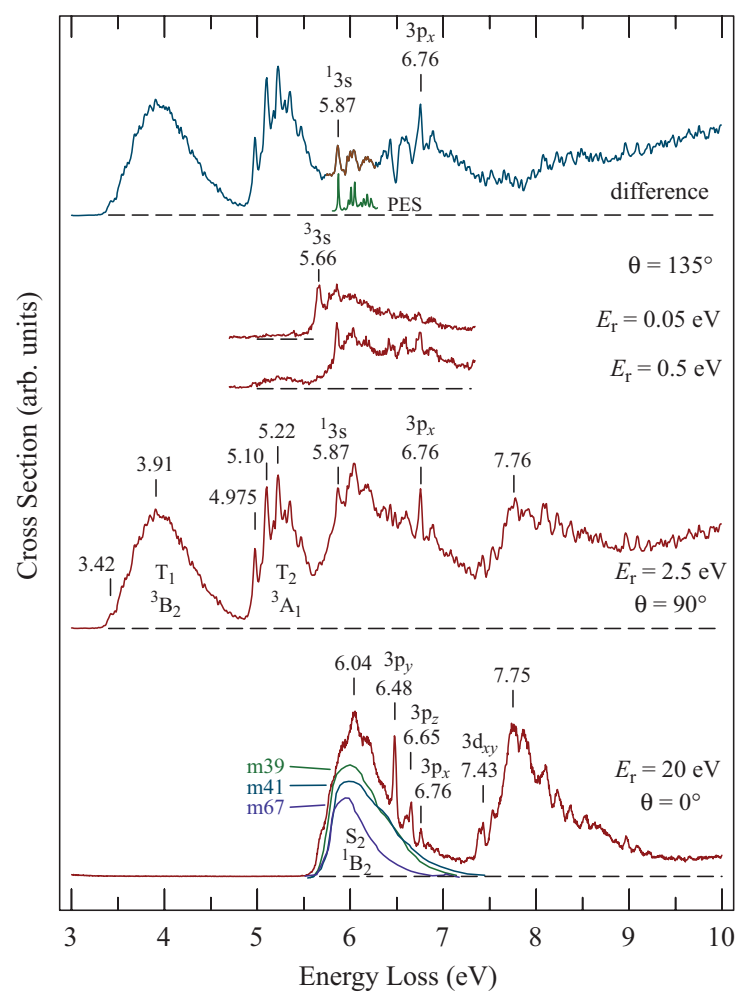

FIG. 3. (Color online) Representative energy-loss spectra emphasizing allowed (bottom spectrum) and forbidden (top four spectra) transitions. They were recorded at the constant residual energies indicated. The topmost spectrum, the difference of the $E_{r}=$ $2.5 \mathrm{eV}, \theta=90^{\circ}$ and the $E_{r}=20 \mathrm{eV}, \theta=0^{\circ}$, enhances the forbidden transitions in the 5.5- to $7.5-\mathrm{eV}$ energy range (the section assigned to the ${ }^{1}\left(\pi_{3}, 3 s\right)$ transition is emphasized by orange [light gray] color). Dissociative attachment spectra from Ref. [16] (p. 114, in arbitrary units) are reproduced under the bottom energy-loss spectrum to illustrate the coincidence in energy and the similarity of band profiles.

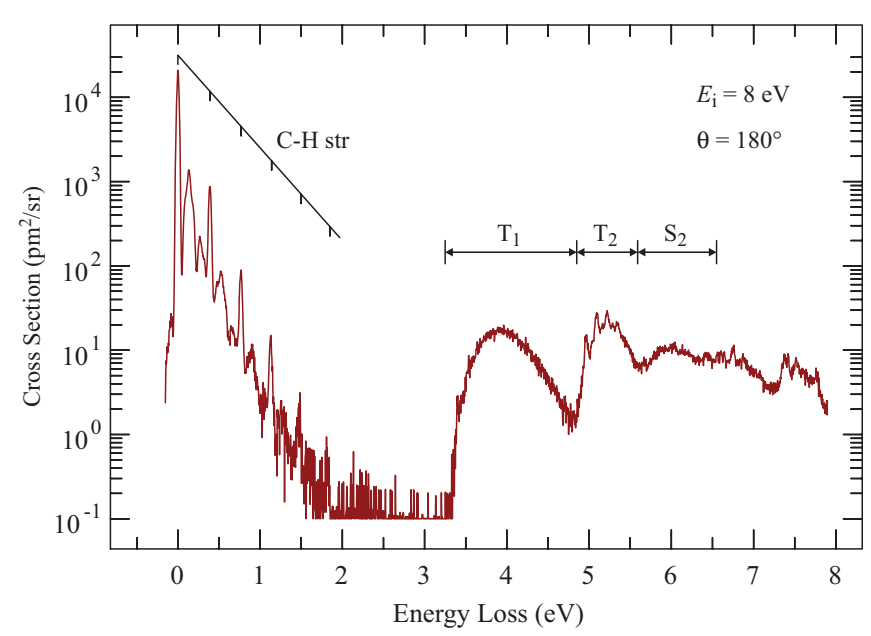

FIG. 4. (Color online) Representative energy-loss spectrum recorded at a constant incident energy.

it is dominated by $S_{2}$ excitation, it is a sum of cross sections for several states. The sections are adjacent, so that the sum of our $T_{1}, T_{2}$, and $S_{2}$ measured cross sections is indicative of all electronic excitations in the energy-loss range 3.25-6.55 eV.

The four singlet states in the $S_{2}$ section are the dipole forbidden $S_{1}{ }^{1} A_{2}(3 s)$ Rydberg state, the $S_{2}$ valence ${ }^{1} B_{2}(V)$ state, which carries the majority of the oscillator strength and is responsible for most of the cross section (perhaps except very near threshold), and the ${ }^{1} B_{1}\left(3 p_{y}\right)$ Rydberg and the ${ }^{1} A_{1}\left(V^{\prime}\right)$ valence state with only a small oscillator strength (see Köppel et al. [20] and Gavrilov et al. [21]).

Elaborate theoretical studies revealed extensive vibronic coupling and strong nonadiabatic effects in the dynamics of these states. Calculations including the nonadiabatic dynamics were required to reproduce the irregular structure of the diffuse $6.04 \mathrm{eV}$ band [20,22]. Theory has shown that although for the lowest part of the spectrum with the origin of the $S_{1}{ }^{1} A_{2}(3 s)$ Rydberg state (discussed below) the adiabatic approximation can be considered valid, it completely breaks down above the lowest conical intersection, which occurs at $\sim 5.9 \mathrm{eV}$ in the experimental spectrum. Above this region the vibrational levels of the two states undergo strong interactions and mixing such that one can no longer assign them to only one electronic state [22,23], making any attempt for deconvolution of individual states futile in principle.

Several of the stronger and well-known Rydberg states are marked in Fig. 3; see, for example, the analysis of Christiansen and Jørgensen [24] for their assignments.

Forbidden transitions in the $S_{2}$ energy-loss range are revealed by the spectra recorded at low residual energies and large scattering angles shown in the center section of Fig. 3. The signal peaks at $6.04 \mathrm{eV}$ in both the "dipole allowed" and the "forbidden" $\left(E_{r}=2.5 \mathrm{eV}\right)$ spectrum but the vibrational structure is more pronounced in the latter, indicating that forbidden transitions are superimposed on the ${ }^{1} B_{2}$ band. We attempted to extract the forbidden contribution to the $90^{\circ}$ spectrum by subtracting the $20-\mathrm{eV}, 0^{\circ}$ spectrum from it, and the result is shown in the top trace of Fig. 3. The shape of the band which emerged around $6 \mathrm{eV}$ is very similar to the shape of the first photoelectron band $\left(\tilde{X}^{2} A_{2}\right)$, 
also shown in Fig. 3, suggesting a forbidden transition to a Rydberg state, presumably the singlet $3 s$ state ${ }^{1}\left(\pi_{3}, 3 s\right)$. Roebber et al. [25] observed the ${ }^{1}\left(\pi_{3}, 3 s\right)$ state at $5.914 \mathrm{eV}$ in multiphoton ionization spectrum-the peak in Fig. 3 is at $5.87 \mathrm{eV}, 40 \mathrm{meV}$ lower, and this difference is more than the difference between the present energies and the energies from optical work for other Rydberg states, generally 10-20 meV, but there is no credible alternative to this assignment. Note that, as explained by Gromov et al. [23], transitions to certain nontotally symmetric vibrational levels of the ${ }^{1} A_{2}(3 s)$ Rydberg state become allowed by intensity borrowing from the ${ }^{1} B_{2}(V)$ state and are responsible for the low-energy features of the 6-eV band in the UV and also the present spectrum recorded with $E_{r}=20 \mathrm{eV}$ at $0^{\circ}$. The band profile of this contribution is not like that of the photoelectron band, however. The band profile in the top spectrum of Fig. 3 is not due to intensity borrowing but to direct excitation by electron impact, and the band profile agrees to that calculated for this case by Gromov et al. [23] and shown in their Fig. 6.

In the spectrum recorded with $E_{r}=0.05 \mathrm{eV}$ we confirm the observation of Asmis [10] of a peak at $5.66 \mathrm{eV}$. Asmis proposed an assignment to a ${ }^{2}\left(\pi_{3}, 3 s^{2}\right)$ Feshbach resonance decaying by an emission of a near-zero-eV electron. We favor an assignment as the ${ }^{3}\left(\pi_{3}, 3 s\right)$ Rydberg state because we fail to observe the peak moving to the left on an energy-loss scale with increasing residual energy, as a peak due to a resonance, locked to a given incident energy, should [26]. The assignment of the 5.66-eV peak as the ${ }^{3} A_{2}{ }^{3}\left(\pi_{3}, 3 s\right)$ Rydberg state is in excellent agreement with the value of $5.77 \mathrm{eV}$ calculated by Bousquet et al. [27] and the value of $5.69 \mathrm{eV}$ calculated by Gavrilov et al. [21]. This assignment also supports the conclusion of Palmer et al. [18], who interpreted a downward shift of the 6.04-eV band at their spectra recorded with a trap depth of $0.1 \mathrm{eV}$ (i.e., low residual energy) as due to the ${ }^{3}\left(\pi_{3}, 3 s\right)$ state, which they placed at $5.8 \mathrm{eV}$.

Note that the $T_{2}$ state is virtually absent in the $E_{r}=0.05 \mathrm{eV}$ spectrum and relatively weak in the $E_{r}=0.5 \mathrm{eV}$ spectrum. This indicates a cross section which rises only gradually above threshold, as will be seen in detail in Sec. IIIC1. In contrast, the ${ }^{3}\left(\pi_{3}, 3 s\right)$ state is seen clearly only very close to threshold, a behavior common for triplet Rydberg states [26].

In accordance with Palmer et al. [18] we see evidence for a broad (presumably valence), forbidden (presumably triplet) state at $6.5 \mathrm{eV}$, seen as a shoulder in the $E_{r}=2.5 \mathrm{eV}$ spectrum and as a broad band (superimposed by sharp Rydberg peaks) in the difference spectrum in Fig. 3.

The narrow peak at $6.76 \mathrm{eV}$, assigned as $3 p_{x}$ [24], is about $4 \times$ weaker than the $3 p_{y}$ state at $6.48 \mathrm{eV}$ in the $\theta=0^{\circ}, E_{r}=$ $20 \mathrm{eV}$ spectrum, in agreement with the calculated oscillator strengths of 0.015 and 0.035 , respectively [24]. In the $\theta=90^{\circ}$, $E_{r}=2.5 \mathrm{eV}$ spectrum, however, the intensities reverse and the $3 p_{x}$ state is much stronger than $3 p_{y}$, which would indicate a forbidden character; we do not have an explanation for this observation.

\section{Cross sections for electronic excitation}

\section{Dependence on electron energy}

Next we present the cross sections for electronic excitation, in the energy-loss ranges given in the preceding section, as a function of electron energy. As in our previous work, they were recorded in two steps. First, energy-loss spectra were recorded at the constant incident electron energies of 5, 6.6, 10, and $20 \mathrm{eV}$, they were corrected for the analyzer response function, and the area under the elastic peak was normalized to the elastic cross section determined in Sec. III A. Figure 4 shows an example of such an energy-loss spectrum (except that, for better readability of the figure, the height of the elastic peak, not the area under it, was normalized to the absolute cross section). Note that the spectrum covers a dynamic range of 5 orders of magnitude. Integrals of the signal in the $T_{1}, T_{2}$, and $S_{2}$ ranges yield the desired inelastic differential cross section.

The energy-loss spectra also cover the $\Delta E$ range with vibrational excitations, with a long progression of the $\mathrm{C}-\mathrm{H}$ stretch vibration, which is doubtlessly excited by a very broad $\sigma^{*}$ resonance found around $8 \mathrm{eV}$ in all hydrocarbons and generally in all molecules with $\mathrm{C}-\mathrm{H}$ bonds. The vibrational energy-loss part of the spectrum permits determination of vibrational excitation cross sections, all vibrational modes included, which will be useful in deriving the grand total cross section below.

In a second step excitation functions were measured at the energy losses corresponding to peaks of the energy-loss spectra in Fig. 3, 3.91, 5.22, and $6.04 \mathrm{eV}$. The excitation functions, corrected for the analyzer response function, were normalized to the differential cross sections determined by integration under the energy-loss bands. The procedure thus involves a slight approximation in that the shape of the excitation function reflects the behavior of the peak energy-loss signal, but it is normalized to the area under the energy-loss signal. As in the case of the angular distributions of the elastic signal in Fig. 1, the discrete absolute values determined from the peak areas are also shown in the figures as circles, as a check of consistency.

The resulting curves are shown in Figs. 5-9. The $S_{2}$ cross section at $\theta=0^{\circ}$ in Fig. 5 rises nearly linearly with energy and attains high values, as expected for a dipole-allowed transition. A similar cross section was obtained in our recent study for a dipole-allowed transition in $\mathrm{Pt}\left(\mathrm{PF}_{3}\right)_{4}$ [28]. The excitation functions of the $T_{1}$ and $T_{2}$ states show clear resonant structure. In the $T_{1}$ case the visibility of the resonant structure is best at $0^{\circ}$, in Fig. 5. The exact position of the two resonances depends to some degree on the angle of observation. The reason for this could be interference of the resonant excitation amplitude with the background amplitude, or that more than two resonances are present, with different angle-dependent intensities.

The present data are compared to those of da Costa et al. [3] at $90^{\circ}$, in Fig. 7. The agreement is excellent at 10 and $15 \mathrm{eV}$. The present data are smaller in the experimentally difficult region near threshold.

\section{Dependence on scattering angle}

To obtain angular distributions of the inelastic cross sections, electron energy-loss spectra similar to those shown in Fig. 4 were recorded, in a repetitive scan to reduce the effects of drifts, for a large number of angles (with $2.5^{\circ}$ increments), controlled by the magnetic angle changer, in $\pm 45^{\circ}$ intervals around the mechanically set analyzer positions of $45^{\circ}, 90^{\circ}$, and $135^{\circ}$. The areas under the electronic energy-loss bands were then determined; the resulting angular distributions were 


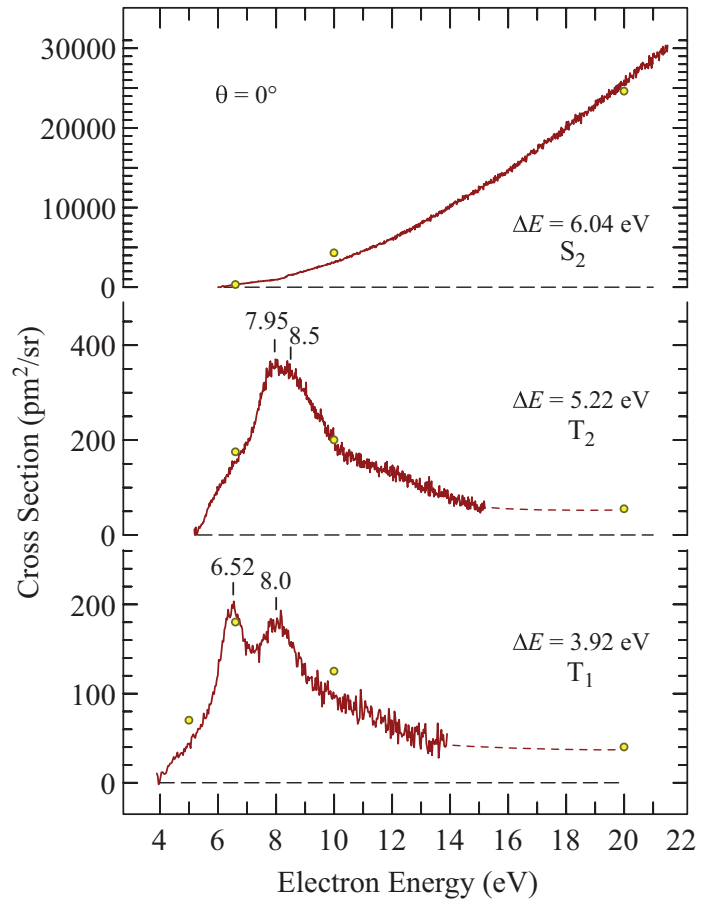

FIG. 5. (Color online) Cross sections for electronic excitation at $0^{\circ}$.

corrected for the angular response functions determined on helium and pieced together to cover the entire $0-180^{\circ}$ angular range. Finally, the resulting shapes were normalized to the absolute cross-sectional values obtained in Sec. IIIC1.

The results are shown in Fig. 10. At $10 \mathrm{eV}$, the present data agree in a perfect way with the experimental data of Khakoo

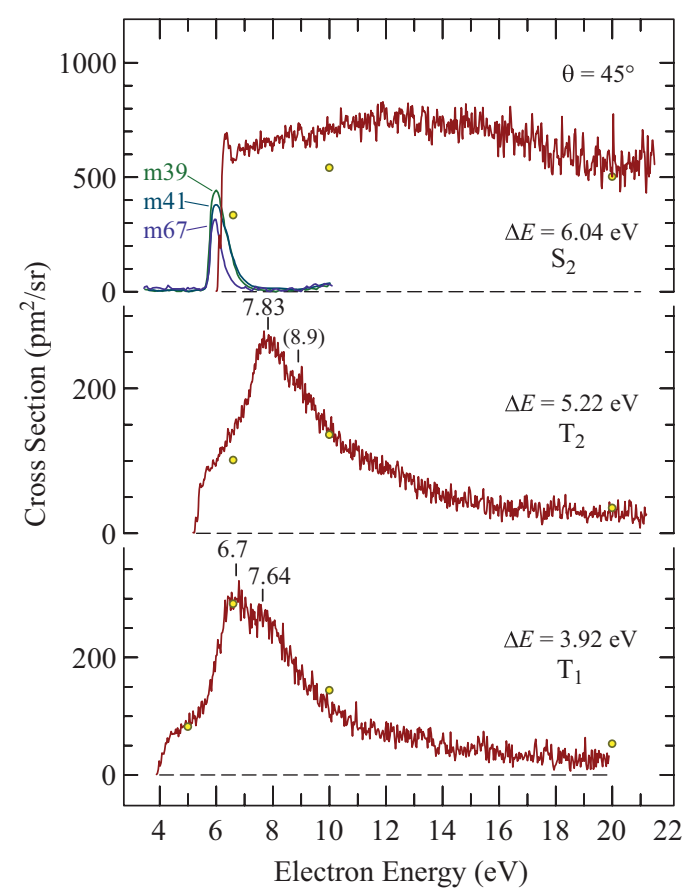

FIG. 6. (Color online) Cross sections for electronic excitation at $45^{\circ}$. Dissociative attachment spectra of Bulliard [16] (p. 114; not in absolute units) are reproduced under the top spectrum.

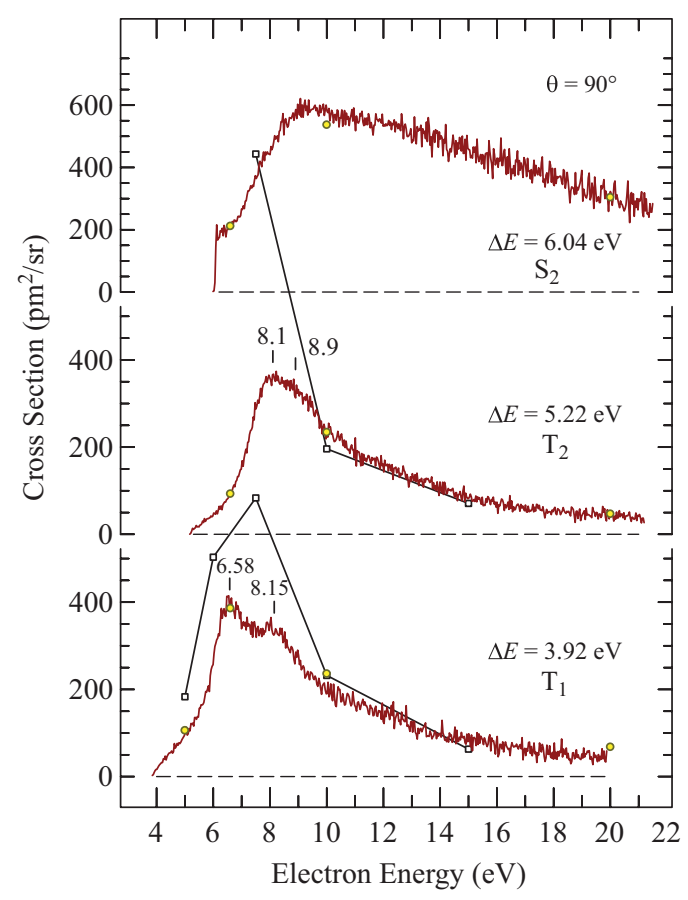

FIG. 7. (Color online) Cross sections for electronic excitation at $90^{\circ}$. Squares show data from Ref. [3].

et al. [3]. Near threshold, the cross section is nearly isotropic for the $T_{1}$ state (at $5 \mathrm{eV}$ ) and slightly forward enhanced for the $T_{2}$ state (at $6.6 \mathrm{eV}$ ). At higher energies, 10 and $20 \mathrm{eV}$, both triplet cross sections are backward enhanced. The general pattern is thus the same as in the prototype case ethene, where the triplet cross section is slightly forward peaked near threshold and backward peaked at $15 \mathrm{eV}$.

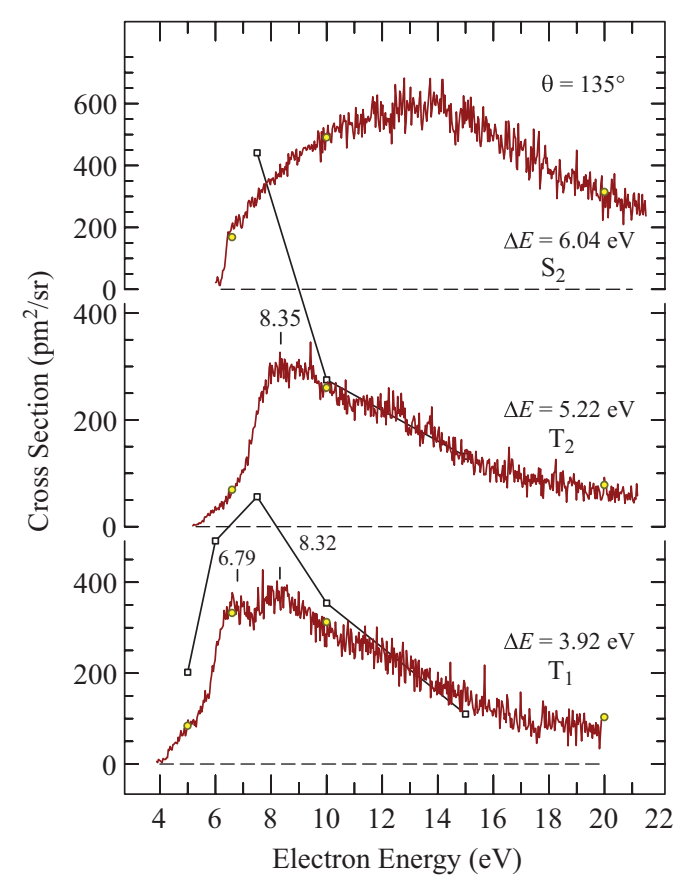

FIG. 8. (Color online) Cross sections for electronic excitation at $135^{\circ}$. Squares show $130^{\circ}$ data from Ref. [3]. 


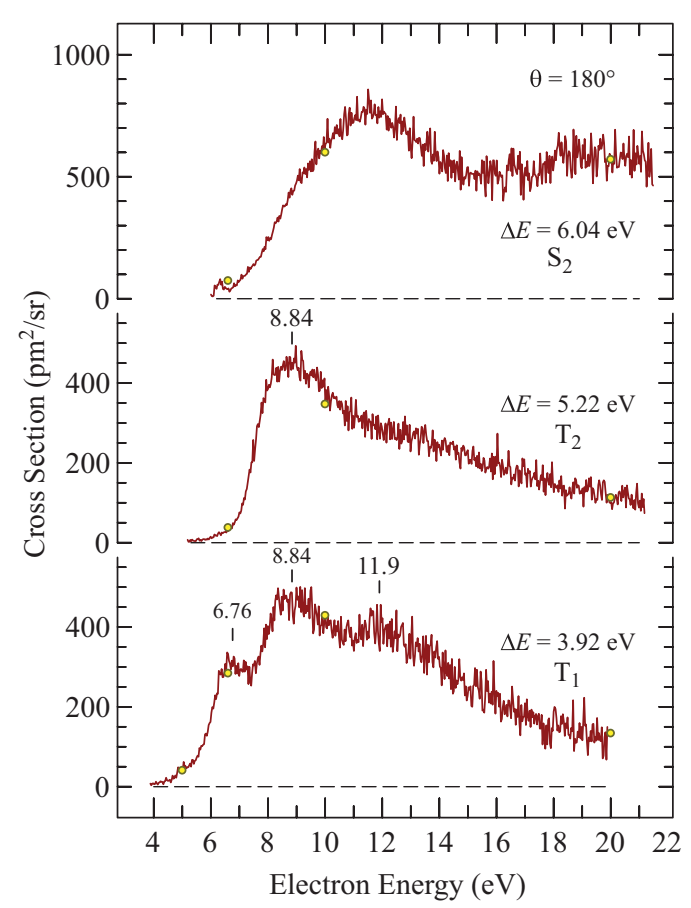

FIG. 9. (Color online) Cross sections for electronic excitation at $180^{\circ}$.

The singlet cross section is also nearly isotropic near threshold (at $6.6 \mathrm{eV}$ ) and has a narrow intense forward peak at higher energies, as expected for a dipole-allowed transition. (Note that the $0^{\circ}$ peak is shown divided by 10 at $10 \mathrm{eV}$ and divided by 100 at $20 \mathrm{eV}$.)

\section{Integral and grand total cross sections}

Integral cross sections were obtained by numerical integration under the angular distributions, and the results are given in Table II. An excellent agreement with the data of Khakoo and coworkers is found at $10 \mathrm{eV}$. The shapes of the cross sections as a function of energy from Figs. 5-9 were then summed, with appropriate weights, as described in Ref. [11], to obtain

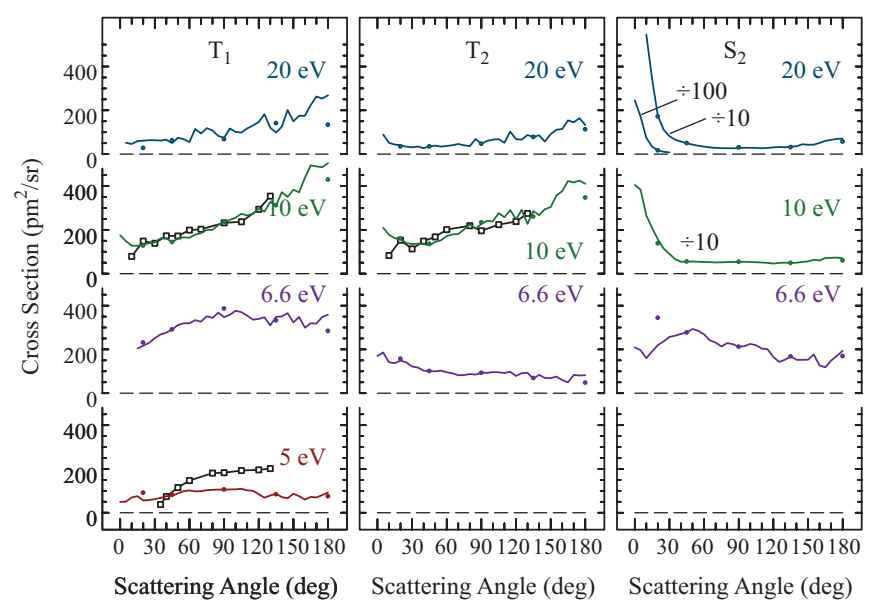

FIG. 10. (Color online) Angular distributions of electronic excitation. Squares show experimental data from Ref. [3].
TABLE II. Integral cross sections for electronic excitation, in $\AA^{2}$; the confidence limit of the present data is $\pm 25 \%$.

\begin{tabular}{lcccc}
\hline \hline Energy & $5.0 \mathrm{eV}$ & $6.6 \mathrm{eV}$ & $10 \mathrm{eV}$ & $20 \mathrm{eV}$ \\
\hline$T_{1}$ & 0.113 & 0.412 & 0.304 & 0.135 \\
Ref. [3] & 0.202 & $(0.581)$ & 0.314 & \\
$T_{2}$ & & 0.117 & 0.283 & 0.075 \\
Ref. [3] & & & 0.273 & \\
$S_{2}$ & & 0.268 & 0.776 & 0.702 \\
\hline \hline
\end{tabular}

integral cross sections as a function of energy. They are shown in Fig. 11. As with the differential data, the present integral data agree very well with those of Khakoo et al. [3] at 10 and $15 \mathrm{eV}$, but are smaller in the experimentally difficult region near threshold.

The theoretical results, shown in Figs. 8 and 9 of the paper of da Costa et al. [3], are burdened by a number of high, generally sharp peaks which are known to be caused by the presence of states that become energetically accessible but were treated as closed channels in the calculations [15]. Since they are known to be spurious, we removed them for the purpose of the comparison in Fig. 11. Qualitative agreement is then obtained in the near-threshold region, in the sense that the calculation reproduces the gradual rise and the shoulder-shape in the first about $3 \mathrm{eV}$ above threshold, followed by a resonance around $8 \mathrm{eV}$. As for the $S_{2}$ state, the present data are lower than the calculation of da Costa et al. [29], although the comparison is only approximate - the present data include all transitions in the $S_{2}$ energy-loss range of Fig. 4 .

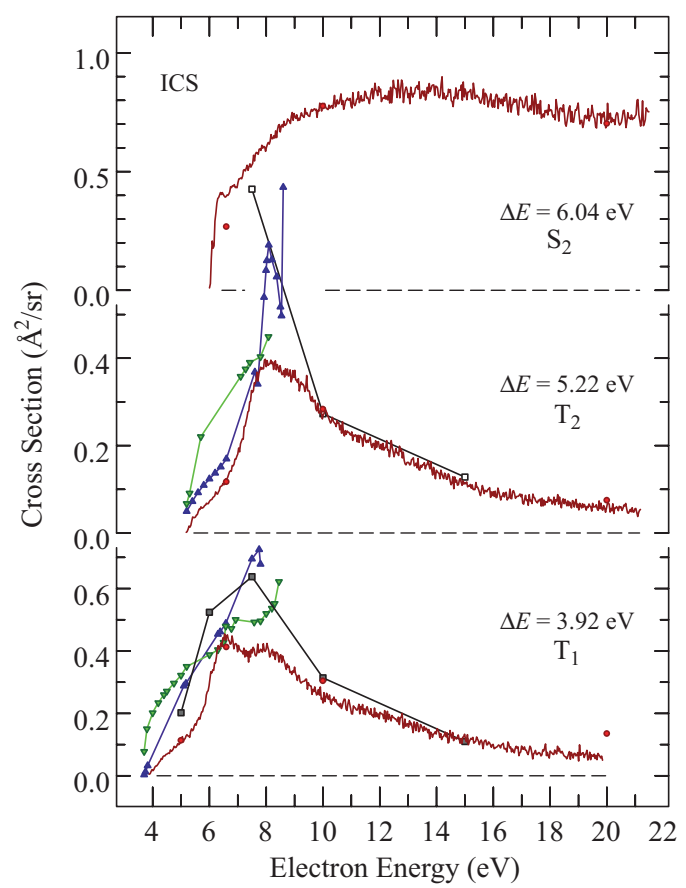

FIG. 11. (Color online) Integral cross sections for electronic excitation. Squares denote the experimental data and triangles the theoretical data of Ref. [3]. (Blue [gray] upward-pointing triangles model 1, and green [gray] downward-pointing triangles model 2 for $T_{1}$ and model 3 for $T_{2}$.) 
TABLE III. Grand total cross section at $10 \mathrm{eV}$, determined as the sum of the elastic, vibrational excitation, electronic excitation, and ionization cross sections, in $\AA^{2}$. The ionization cross section is taken from Ref. [5].

\begin{tabular}{ccccccc}
\hline \hline Energy & Elastic & VE & EE & Ionization & Total & Ref. [5] \\
\hline $10 \mathrm{eV}$ & 32.2 & 2.92 & 3.13 & 0.185 & 38.4 & 48.95 \\
\hline \hline
\end{tabular}

The present data were combined in Table III to yield the grand total cross section at $10 \mathrm{eV}$ and to permit comparison with the data of Szmytkowski et al. [5]. The VE cross section includes all vibrational modes, and the EE cross section includes all electronic states accessible at an incident electron energy of $10 \mathrm{eV}$. The ionization cross section, not measured in this work, was taken from Szmytkowski et al. [5]. The present grand total cross section obtained in this way is $22 \%$ lower than that of Szmytkowski et al. [5]. The confidence limit of the data of Szmytkowski et al. is indicated as $\pm 6 \%$. The present confidence limit is $\pm 15 \%$ for the elastic cross sections and $\pm 25 \%$ for the inelastic cross sections. The grand total cross section is dominated by elastic scattering and its confidence limit is thus only slightly larger than $\pm 15 \%$. The agreement of the present data and that of Szmytkowski et al. is thus on the borderline of the combined error limits. This is surprising because a similar past comparison of data from the same two laboratories, for carbon monoxide, yielded an excellent agreement [11].

\section{E. Assignment of resonances}

Figure 12 illustrates the resonant structures of ethene and furan by representative vibrational and electronic excitation spectra and points out the similarity between the two compounds. The spectra of the two compounds are in a strikingly simple relation-one $\mathrm{C}=\mathrm{C}$ bond in ethene results in one shape and one core-excited resonance, two $\mathrm{C}=\mathrm{C}$ bonds in furan result in two shapes and two core-excited resonances. Based on this observation and following the conclusions of Asmis [10] we propose an interpretation of the resonances based on the assignment in ethene. The assignment in ethene, illustrated in Fig. 13, stipulates that the shoulder in the 4.5- to $6-\mathrm{eV}$ range in the $T_{1}$ excitation cross section is caused by a high-energy tail of the ${ }^{2}\left(\pi^{*}\right)$ shape resonance and the $7-\mathrm{eV}$ band to the ${ }^{2}\left(\pi, \pi^{* 2}\right)$ core excited shape resonance [2,30]. (We label shape resonances as $\tilde{s}_{n}$ and valence core-excited shape resonances as $\tilde{c}_{n}$.) It should be recalled that one-electron processes are prominent in the formation and decay of resonances; that is, resonances decay predominantly into their parent states. The prominent role of the core-excited shape resonance in Fig. 13 is understandable because a departure of an electron from the $\pi^{*}$ orbital leads directly to the $T_{1}{ }^{3}\left(\pi, \pi^{*}\right)$ triplet excited state.

The parent state of the ${ }^{2}\left(\pi^{*}\right)$ shape resonance is primarily the ground electronic state of ethene, because the formal removal of the $\pi^{*}$ electron leads to the ground electronic configuration. The ${ }^{2}\left(\pi^{*}\right)$ shape resonance expectedly decays primarily to the electronic ground state, often accompanied by vibrational excitation, which was used to detect it in Fig. 12. Less conventionally, the $T_{1}^{3}\left(\pi, \pi^{*}\right)$ triplet excited state can

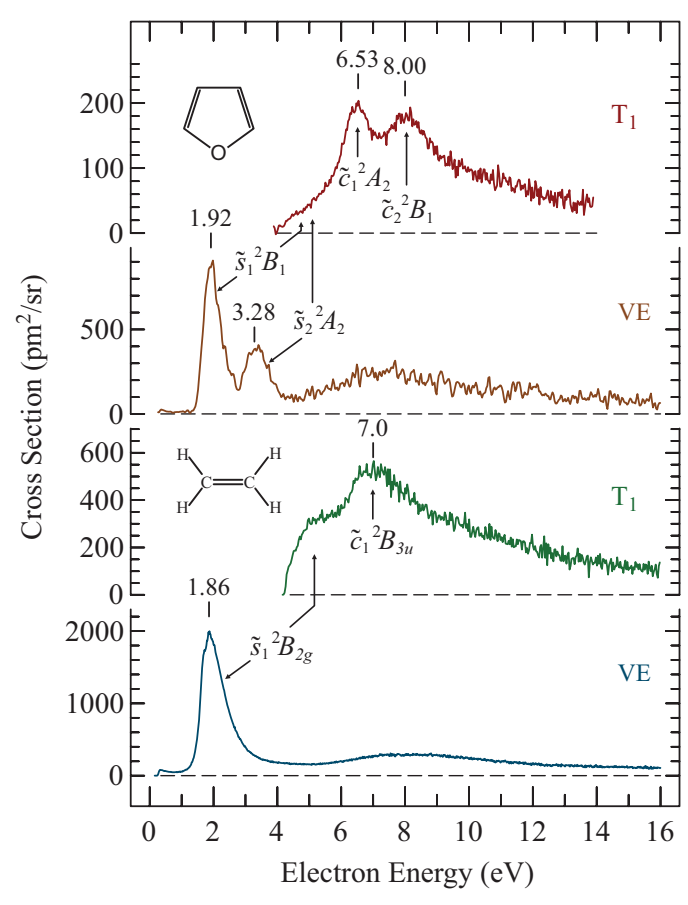

FIG. 12. (Color online) Comparison of representative spectra of ethene (bottom, from Ref. [2]) and furan (top). Vibrational excitation cross sections reveal primarily shape resonances; electronic excitation cross sections reveal primarily core-excited shape resonances.

also be considered to be the parent state of the ${ }^{2}\left(\pi^{*}\right)$ shape resonance because removal of a $\pi$ electron leads to the $T_{1}$ state (Fig. 13). One would initially not expect the ${ }^{2}\left(\pi^{*}\right)$ shape resonance to play a role in the $T_{1}$ excitation because the resonance is energetically below the $T_{1}$ state, but calculations have shown that the finite width of the shape resonance leads to a high-energy tail which may dominate the excitation of the $T_{1}$ state, although the resonance lies nominally below the $T_{1}$ threshold. This excitation mechanism has been identified quite early in the case of $\mathrm{O}_{2}$ by Gauyacq and coworkers [31,32]. The role of low-lying shape resonances on electronic excitation has been studied $a b$ initio for both ethene [30] and furan [8].

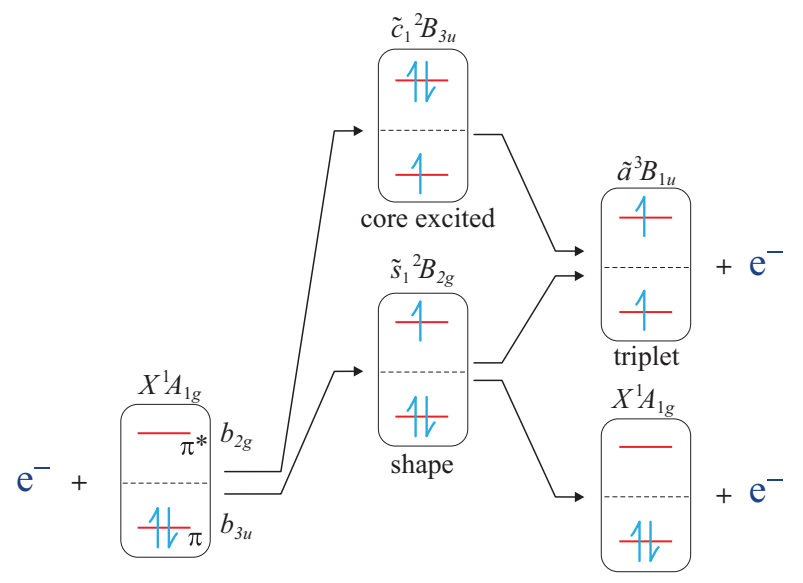

FIG. 13. (Color online) Configurations of ethene and its shape and core-excited resonances. Arrows indicate parentage relations, i.e., the excitation pathways. 


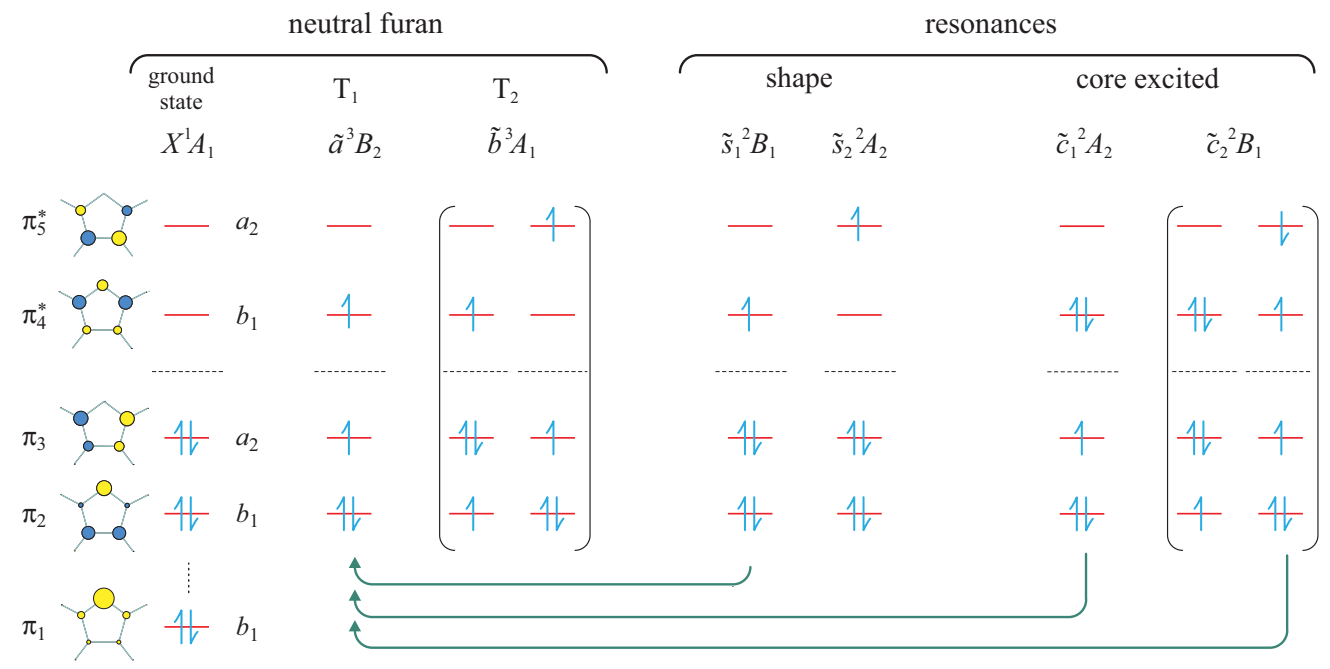

FIG. 14. (Color online) Configurations of furan and its shape and core-excited resonances. Parentheses indicate that both configurations contribute to a given state. The curved green (gray) arrows at the bottom indicate parentage relations; i.e., they indicate for each resonance into which excited states of neutral furan it is expected to decay by formal removal of only one electron.

We now investigate whether these general lines can be applied to the excitation of the $T_{1}$ state of furan, by regarding the parentage relations in Fig. 14. The two shape resonances result from temporary occupation of the $b_{1} \pi_{4}^{*}$ and $a_{2} \pi_{5}^{*}$ orbitals, respectively. These orbitals are in a first approximation the in-phase and out-of-phase combinations of the $\mathrm{C}=\mathrm{C} \pi^{*}$ orbitals and one may initially expect their energies to be arranged symmetrically around the energy of the ${ }^{2}\left(\pi^{*}\right)$ shape resonance of ethene. This is clearly not the case- - the ${ }^{2}\left(\pi_{4}^{*}\right) \tilde{s}_{1}^{2} B_{1}$ resonance is higher than expected from a symmetrical split. This can be rationalized as a result of conjugative destabilization of the symmetric combination of the $\mathrm{C}=\mathrm{C} \pi *$ orbitals by the $p_{\pi}$ "nonbonding" orbital on the $\mathrm{O}$ atom, shown at the bottom of Fig. 14. (This conjugative destabilization is clearly manifested in methyl vinyl ether, $\mathrm{CH}_{2}=\mathrm{CHOCH}_{3}$, when one hydrogen of ethene is replaced by a methoxy group, which pushes the shape resonance up by $0.35 \mathrm{eV}$ to $2.2 \mathrm{eV}$ and the core-excited resonance up by $0.5 \mathrm{eV}$ to $7.5 \mathrm{eV}$ [16].)

Figure 14 shows that the first shape resonance, $\tilde{s}_{1}{ }^{2} B_{1}$, is a daughter state of the $T_{1}$ state, in the same somewhat unorthodox sense as in ethene, because a formal removal of the $a_{2} \pi_{3}$ electron leads to the $T_{1}$ configuration. The high-energy tail of the first shape resonance is thus expected to excite the $T_{1}$ state as in ethene and we assign the shoulder at $4-5.5 \mathrm{eV}$ in the corresponding cross section (for example, the top curve in Fig. 12) in part to the $\tilde{s}_{1}{ }^{2} B_{1}$ shape resonance.

The $T_{1}$ triplet state $\tilde{b}^{3} B_{2}$ is clearly a parent state of the first core-excited shape resonance $\tilde{c}_{1}{ }^{2} A_{2}$, because removal of a $b_{1} \pi_{4}^{*}$ electron from the resonance leads to the $T_{1}$ configuration. The $\tilde{c}_{1}{ }^{2} A_{2}$ resonance is thus expected to decay efficiently to the $T_{1}$ state and we assign the $6.53-\mathrm{eV}$ peak in the corresponding cross section (Fig. 12) to this resonance.

This resonance has the same $\left(a_{2}\right)$ symmetry as the second shape resonance, and the second shape resonance will thus acquire some core-excited character by configuration mixing. This core-excited admixture will permit the second shape resonance $\tilde{s}_{2}$ to also contribute to the excitation of the $T_{1}$ state.
Note that mixing of shape and core-excited configurations has been invoked early by Nenner and Schulz in benzene and aromatic heterocycles [33] and has been confirmed experimentally in benzene by observing the decay of the third, nominally shape, resonance at $4.8 \mathrm{eV}$ into the lowest triplet state [26], and finally proven by elaborate $a b$ initio scattering calculations in pyrazine by Winstead and McKoy [34]. We thus conclude that both the first and the second shape resonances in furan can decay into the first triplet state $T_{1}$ by different mechanisms and thus contribute to the shoulder in the cross section in the 4- to $5.5-\mathrm{eV}$ range.

Two configurations may be proposed for the second coreexcited resonance. Taking the first core-excited resonance as a departing point, one may either move the hole one orbital down or move one of the temporarily captured electrons one orbital up. Both configurations obtained in this way have the $b_{1}$ symmetry and both have similar energies, because the separations of the $\pi$ and the $\pi^{*}$ orbitals are about the same in furan. Configuration interaction (CI) will come into play and both configurations will be mixed in the $\tilde{c}_{2}{ }^{2} B_{1}$ core-excited shape resonance, as indicated by the parentheses in Fig. 14. The $T_{1}$ state is a parent state of one of these configurations, the one on the right in the parentheses, the $\tilde{c}_{2}{ }^{2} B_{1}$ core-excited shape resonance is expected to decay efficiently into the $T_{1}$ state, and we assign the $8.0-\mathrm{eV}$ peak in the corresponding cross section (Fig. 12) to this resonance.

Two configurations contribute substantially to the second triplet state $\tilde{b}^{3} A_{1}$. One of them is a parent state of the second shape resonance $\tilde{s}_{2}{ }^{2} A_{2}$, which is thus presumably responsible for the shoulder in the 5.5- to $7-\mathrm{eV}$ range in the cross section for $T_{2}$ excitation; see, for example, Fig. 5. One of the configurations of the $T_{2}$ state is also a parent state of one of the configurations of the second core-excited resonance ${ }^{2} B_{1}$ and we therefore assign, in accordance with Asmis [10], the 7.95-eV peak in the $T_{2}$ cross section (see, for example, the center curve in Fig. 5) to this resonance. (The two peaks in the $T_{2}$ excitation functions are strongly overlapping. They are best distinguishable in the excitation functions recorded at $0^{\circ}$, and 
they can also be clearly distinguished in the $0^{\circ}$ spectra recorded with the magnetically collimated spectrometer by Asmis [10].) Asmis [10] performed semiempirical intermediate neglect of differential overlap, parametrized for spectroscopy (INDO/S) CI calculations on the furan anion and the results agree with the above qualitative reasoning. Although semiempirical calculations are now obsolete and lack proper scattering boundary conditions, they provided the proper insight. The same conclusion can be reached from more modern timedependent density functional theory (TD-DFT) calculations on furan anion, which we carried out but do not present here because they also lack proper coupling to the continuum and did not yield any new insight.

\section{F. Relation to dissociative electron attachment}

The core-excited shape resonances seen in the present cross sections for electronic excitation do not appear in dissociative attachment spectra reported by Sulzer et al. [9] and by Bulliard [16] (p. 114); the autodetachment rate is apparently too fast to permit chemistry on the resonant potential energy surfaces. Instead, the DEA spectra (yields of fragments with masses 39, 41, and 67) are dominated by a band which is nearly identical to the 6.04-eV energy-loss band both in terms of energy and shape, as can be seen in Fig. 3. DEA in furan thus seems to be dominated by a threshold resonance where an electron is weakly bound to the $S_{2}{ }^{1} B_{2}$ valence excited state. The resonance could be designated as $\tilde{x}^{2} B_{2}$, with the configuration ${ }^{2}\left(\pi_{3}, \pi_{4}^{*} \bar{s}\right)$, where $\bar{s}$ denotes a weakly bound electron in an $s$-like orbital. This process is common in organic molecules with $\pi$ bonds and consequently low-lying valence excited states where a DEA band nearly coinciding with the lowest valence singlet excited state is often observed, assigned to a resonance where the incoming electron is temporarily captured in a diffuse orbital around a valence excited core. Systematic evidence for such states was collected by the group of Khvostenko [35]. We have observed this type of resonances in many compounds, for example, in $\mathrm{CS}_{2}$ [36], methyl vinyl ether ([16], chapter IV.1), and 2-chlorobenzyl bromide ([16], p. 102) (see also chapter IV.5 of Ref. [16]).

Somewhat surprisingly, there is no clear evidence for this resonance in the cross section for excitation of its parent, the valence $S_{2}{ }^{1} B_{2}(V)$ state, where it could be expected to cause a threshold peak in the excitation function. It could be, however, that the fact that at nonzero scattering angles the onset of the $S_{2}$ excitation function is vertical, stepwise, not gradual like that of the triplet states, as can be seen for example in Fig. 6, and is a manifestation of the $\tilde{x}^{2} B_{2}$ resonance. (The fact that the DEA onset appears slightly under the $S_{2}$ excitation onset in Fig. 6 is the consequence of the $S_{2}$ excitation function being measured at the peak of the $S_{2}$ energy-loss band at $6.04 \mathrm{eV}$, whereas already the lowest vibrational level of the $S_{2}$ state contributes to DEA as can be seen in Fig. 3.)

It is interesting to speculate that the efficiency of this resonance for DEA is given in part (apart from a sufficiently slow autodetachment rate) by its potential surface having similar complexity as that of its parent state, recently revealed by high-level calculations $[22,37]$ with vibronic coupling and conical intersections leading to repulsive sections of potential energy surfaces and to chemical change.

\section{CONCLUSIONS}

Elastic and electronic excitation cross sections were measured as a function of both electron energy and scattering angle. For elastic scattering very good agreement was found with the measurements of Khakoo et al. [4], both for the the angular distributions of the cross sections (Fig. 1) and the differential elastic cross sections plotted as a function of energy at $45^{\circ}$ and $90^{\circ}$ (Fig. 2). Very good agreement was also found for the magnitudes of the integral elastic cross sections (Table I). For the excitation of the $T_{1}\left({ }^{3} B_{2}\right)$ and $T_{2}\left({ }^{3} A_{1}\right)$ triplet states, very good agreement was found with the experiment of Khakoo et al. at 10 and $15 \mathrm{eV}$ (Figs. 7 and 8), but the present cross sections are smaller near threshold. The same is true for the integral cross sections (Fig. 11).

Concerning comparison with theoretical results, for elastic cross sections very good agreement was found with the calculated elastic cross sections of da Costa et al. [3] in terms of the angular distributions of the differential cross sections (Fig. 1) and the magnitudes of the integral elastic cross sections (Table I). The situation is more complicated for inelastic cross sections, where the calculation is burdened by spurious sharp peaks. When these peaks are disregarded, qualitative agreement is obtained in the near-threshold region, but the core-excited resonances of the experimental spectrum are not reproduced in detail by the calculation (Fig. 11).

The shapes of the inelastic cross sections as a function of energy reveal clear resonant structure and confirm the (not absolute) observations and conclusions of Asmis [10]. A clear resemblance is found between ethene and the present cyclic diene, indicating very related excitation mechanisms. Based on this resemblance, parentage relations, and reference to existing theory, an assignment of two resonances in the excitation function of the lowest triplet state is proposed as the core-excited valence shape resonances $\tilde{c}_{1}{ }^{2} A_{2}$ at $6.5 \mathrm{eV}$ and $\tilde{c}_{2}{ }^{2} B_{1}$ at $8.0 \mathrm{eV}$ (the positions of the peaks depend slightly on the angle of observation). The first of these resonances is proposed to be mixed with the second shape resonance of the same symmetry, and the second is proposed to be a mixture of the ${ }^{2}\left(\pi_{2}, \pi_{4}^{* 2}\right)$ and ${ }^{2}\left(\pi_{3}, \pi_{4}^{*} \pi_{5}^{*}\right)$ configurations. Two core-excited shape resonances, slightly less pronounced, are observed at about 8.0 and $8.7 \mathrm{eV}$ in the excitation function of the second triplet state (the exact positions of the peaks also depend on the angle of observation). The first is assigned as the same $\tilde{c}_{2}{ }^{2} B_{1}$ resonance seen in the excitation function for the $T_{1}$ state. These assignments are in agreement with similar reasoning and semiempirical CI calculations of anion excited states by Asmis.

Bulliard [16] has shown that the pattern observed for ethene is also found for methoxy substituted ethene methyl vinyl ether, with energy shifts of the ${ }^{2}\left(\pi^{*}\right)$ shape and the ${ }^{2}\left(\pi, \pi^{* 2}\right)$ resonances qualitatively understandable as a consequence of the well-known conjugative destabilization of the $\pi$ and $\pi^{*}$ orbitals by the methoxy group. Asmis [10] has shown that the general pattern described here for furan is also found in other cyclic dienes: cyclopentadiene, thiophene, and pyrrole. 
This shows that the pattern of triplet states being excited by shape resonances near threshold followed by a relatively narrow valence core-excited shape resonances is quite general, independent of the chemical details of the target.

Comparison with existing dissociative electron attachment spectra reveals a threshold resonance which in terms of energy and Franck-Condon profile coincides with the lowest singlet valence excited state $S_{2}{ }^{1} B_{2}$ and must consequently correspond to an electron very loosely bound to the $S_{2}{ }^{1} B_{2}$ core, a ${ }^{2}\left(\pi_{3}, \pi_{4}^{*} \bar{s}\right) \tilde{x}^{2} B_{2}$ resonance. Recent elaborate studies of the ring-opening dynamics on the potential energy surfaces $[22,37]$ of the parent state reveal a great complexity of the process caused by extensive vibronic coupling and numerous conical intersections. It is very likely that similar complexity is found on the potential energy surfaces of the core-excited shape resonance surface responsible for DEA. It would be most interesting and intriguing to see whether the dynamics on the threshold resonance could be treated by the theoretical methods developed for the singlet excited state chemistry. The effort would also be desirable in the light of the quest for understanding electron-driven chemistry.
A general conclusion is that theoretical description of electronic excitation of polyatomic molecules is not yet a solved problem. In view of the central importance of electronic excitation for electron-driven chemistry, further effort towards solving it is very important. Furan is a suitable test case, representing a midpoint between the simplicity of ethene and the complexity of real-life biomolecules or FEBIP precursors. The theoretical methods should be tested not only by their capacity to reproduce the cross-section magnitudes at low energies but also by their capacity to reproduce the resonant structure and elucidate the excitation mechanism.

\section{ACKNOWLEDGMENTS}

We thank Professor Romarly da Costa and Professor Marco Lima for helpful comments and for communicating the results of their calculation in digital form. This research is part of Project No. 200020-144367/1 of the Swiss National Science Foundation and of the COST Action CM1301 CELINA.
[1] M. Allan, Chem. Phys. Lett. 225, 156 (1994).

[2] M. Allan, C. Winstead, and V. McKoy, Phys. Rev. A 77, 042715 (2008).

[3] R. F. da Costa, M. H. F. Bettega, M. A. P. Lima, M. C. A. Lopes, L. R. Hargreaves, G. Serna, and M. A. Khakoo, Phys. Rev. A 85, 062706 (2012).

[4] M. A. Khakoo, J. Muse, K. Ralphs, R. F. da Costa, M. H. F. Bettega, and M. A. P. Lima, Phys. Rev. A 81, 062716 (2010).

[5] C. Szmytkowski, P. Możejko, E. Ptasińska-Denga, and A. Sabisz, Phys. Rev. A 82, 032701 (2010).

[6] L. R. Hargreaves, R. Albaridy, G. Serna, M. C. A. Lopes, and M. A. Khakoo, Phys. Rev. A 84, 062705 (2011).

[7] M. H. F. Bettega and M. A. P. Lima, J. Chem. Phys. 126, 194317 (2007).

[8] R. F. da Costa, M. H. F. Bettega, and M. A. P. Lima, Phys. Rev. A 77, 012717 (2008).

[9] P. Sulzer, S. Ptasińska, F. Zappa, B. Mielewska, A. R. Milosavljević, P. Scheier, T. D. Märk, I. Bald, S. Gohlke, M. A. Huels, and E. Illenberger, J. Chem. Phys. 125, 044304 (2006).

[10] K. Asmis, Ph.D. thesis, University of Fribourg, 1996 http://ethesis.unifr.ch/theses/index.php\#Sciences

[11] M. Allan, Phys. Rev. A 81, 042706 (2010).

[12] A. Gopalan, J. Bömmels, S. Götte, A. Landwehr, K. Franz, M. W. Ruf, H. Hotop, and K. Bartschat, Eur. Phys. J. D 22, 17 (2003).

[13] J. C. Nickel, P. W. Zetner, G. Shen, and S. Trajmar, J. Phys. E: Sci. Instrum. 22, 730 (1989).

[14] R. K. Nesbet, Phys. Rev. A 20, 58 (1979).

[15] R. F. da Costa and M. A. P. Lima (private communication).

[16] C. Bulliard, Ph.D. thesis, University of Fribourg, 1994 http://ethesis.unifr.ch/theses/index.php\#Sciences

[17] W. M. Flicker, O. A. Mosher, and A. Kuppermann, J. Chem. Phys. 64, 1315 (1976).

[18] M. H. Palmer, I. C. Walker, C. C. Ballard, and M. F. Guest, Chem. Phys. 192, 111 (1995).
[19] A. Giuliani and M.-J. Hubin-Franskin, Int. J. Mass Spectrom. Ion Proc. 205, 163 (2001).

[20] H. Köppel, E. V. Gromov, and A. B. Trofimov, Chem. Phys. 304, 35 (2004).

[21] N. Gavrilov, S. Salzmann, and C. M. Marian, Chem. Phys. 349, 269 (2008).

[22] E. V. Gromov, V. S. Reddy, F. Gatti, and H. Köppel, J. Chem. Phys. 139, 234306 (2013).

[23] E. V. Gromov, A. B. Trofimov, N. M. Vitkovskaya, H. Köppel, J. Schirmer, H.-D. Meyer, and L. S. Cederbaum, J. Chem. Phys. 119, 737 (2003).

[24] O. Christiansen and P. Jørgensen, J. Am. Chem. Soc. 120, 3423 (1998).

[25] J. L. Roebber, D. P. Gerrity, R. Hemley, and V. Vaida, Chem. Phys. Lett. 75, 104 (1980).

[26] M. Allan, J. Electron Spectrosc. Relat. Phenom. 48, 219 (1989).

[27] D. Bousquet, R. Fukuda, D. Jacquemin, I. Ciofini, C. Adamo, and M. Ehara, J. Chem. Theo. Comp. 10, 3969 (2014).

[28] M. Allan, J. Chem. Phys. 134, 204309 (2011).

[29] R. F. da Costa, M. H. F. Bettega, M. T. N. Varella, and M. A. P. Lima, J. Phys.: Conf. Ser. 388, 012015 (2012).

[30] R. F. da Costa, M. H. F. Bettega, and M. A. P. Lima, Phys. Rev. A 77, 042723 (2008).

[31] D. Teillet-Billy, L. Malegat, and J. P. Gauyacq, J. Phys. B: At. Mol. Phys. 20, 3201 (1987).

[32] D. Teillet-Billy, L. Malegat, J. P. Gauyacq, R. Abouaf, and C. Benoit, J. Phys. B: At. Mol. Opt. Phys. 22, 1095 (1989).

[33] I. Nenner and G. J. Schulz, J. Chem. Phys. 62, 1747 (1975).

[34] C. Winstead and V. McKoy, Phys. Rev. Lett. 98, 113201 (2007).

[35] V. I. Khvostenko, A. S. Vorob'yov, and O. G. Khvostenko, J. Phys. B: At. Mol. Opt. Phys. 23, 1975 (1990).

[36] R. Dressler, M. Allan, and M. Tronc, J. Phys. B: At. Mol. Phys. 20, 393 (1987).

[37] E. V. Gromov, C. Lévêque, F. Gatti, I. Burghardt, and H. Köppel, J. Chem. Phys. 135, 164305 (2011). 